\title{
Adult-onset pulmonary involvement in Niemann-Pick disease type B
}

\author{
F. Iaselli1, G. Rea22, S. Cappabianca1, G. Fabozzi1, M. Montemarano1, \\ C. Vitale ${ }^{3}$, A.A. Stanziola3, R. Muto², A. Rotondo1
}

ABSTRACT: Adult-onset pulmonary involvement in Niemann-Pick disease type B. F. Iaselli, G. Rea, S. Cappabianca, G. Fabozzi, M. Montemarano, C. Vitale, A.A. Stanziola, R. Muto, A. Rotondo.

Niemann-Pick disease type $B$ is caused by a deficiency in acid sphingomyelinase activity; among the six variants of Niemann-Pick disease known to date, it is the most frequently associated with lung involvement, a major cause of morbidity and mortality in this subtype in patients of all ages. Nevertheless, the vast majority of reports in the literature concern infantile forms, while less reported is, for several reasons, the onset in adults being consequently still poorly understood and characterized its clinical, radiographic and functional manifestations. We re- port a case of a 37 years-old female patient affected by subtype $B$ since she was an infant, operated for aortic valve replacement two years before and came to our attention for the onset of a worsening exertional dyspnoea which proved, through a series of functional tests and radiological exams, to be a consequence of the diffuse lung involvement by the metabolic disorder; we performed a review on this topic through a Medline search of all the available "adult-onset" case reports published since the first description in 1964, also considering the possible association between NPDB and, more generally lysosomal storage disorders, and the valvular disease, already suggested by several Authors in previous works.

Monaldi Arch Chest Dis 2011; 75: 4, 235-240.

Keywords: Niemann-Pick diasease type B, High-Resolution Computed Tomography, Interstitial Disease, Heart Valve Disease.

${ }^{1}$ Department of Clinical Internal Medicine 'F. Magrassi - L. Lanzara', Science Section of Radiology, Seconda Università degli Studi di Napoli, Primo Policlinico di Napoli,

2 Department of Diagnostic Imaging, A.O.R.N. Monaldi, Napoli,

3 Department of Respiratory Medicine, Università Federico II di Napoli, A.O.R.N. Monaldi, Napoli, Italy.

Correspondence: Francesco Iaselli, Corso Trieste 273,81100 Caserta, Italy; e-mail:francescoiaselli@hotmail.it

\section{Introduction}

Niemann-Pick disease (NPD) is a rare inherited autosomal recessive disease characterised by the intracellular accumulation of sphingomyelin (SM) in the liver, spleen, lungs, bone marrow and brain within "foamy" reticuloendothelial cells. According to the affected organs and the clinical severity, the disease is currently classified into six subtypes, from A to F. Subtype B (NPDB) is caused by a deficiency in acid sphingomyelinase (ASM) activity and is characterided by the spur of the nervous system, which is typically involved in Form A (NPDA), by a fair prognosis with survival to adulthood in most cases and by a chronic, polivisceral involvement, slowly and inexorably progressive. Among the six variants known to date, it is the most frequently associated with lung involvement, a major cause of morbidity and mortality in this subtype in patients of all ages. Nevertheless, the vast majority of reports in the literature concern infantile forms, while less reported, for several reasons, is the onset in adults which, as a consequence is still poorly understood and characterised in terms of its clinical, radiographic and functional manifestations. We report a case of a 37 year-old female patient affected by NPDB since she was an infant, operated on for aortic valve replacement two years before and came to our attention for the onset of a worsening exertional dyspnoea which proved, through a series of functional tests and radiological exams, to be a consequence of the diffuse lung involvement by the metabolic disorder. We performed a review on this topic through a Medline search of all the available case reports concerning the adult onset involvement in NPDB published since the first description in 1964, also suggesting a possible association between the valvular disease found in our patient and NPDB or, more generally, lysosomal storage disorders, a hypothesis already put forward by several Authors in previous works.

\section{Case report}

In May 2010, a 37 year-old female patient complaining of a worsening exertional dyspnoea in recent weeks came to our attention. When she was 2 years old, following the discovery of a marked hepatosplenomegaly she was diagnosed with a NPDB; in the following months the polivisceral involvement of the disease progressed with the appearance of emocoagulative and endocrine 
disorders, worsening hepatosplenomegaly, in-

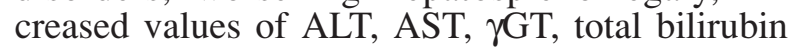
and cholesterol, thrombocytopenia and mild leukopenia and, finally, aortic valve stenosis, mitral stenoinsufficiency and tricuspidal insufficiency. In 2008 the worsening of the stenosis of the aortic valve required an operation to replace it with a biological prosthesis, which was resolved without complications. At the last hospitalisation the recurrent dyspnoea and the presence of bilateral basal rales and crackles at chest examination led us to perform a Chest X-Ray (CXR), which showed the presence of a bilateral reticular interstitial thickening more severe at lower lobes, suggestive of a diffuse infiltrative disease. Therefore, in order to confirm our suspicion, we performed a High Resolution Computed Tomography (HRCT) exam which showed thickened interlobular and intralobular septa associated with bilateral "ground glass" opacities; where intermixed these two features created the typical "crazy paving" pattern. All these radiographic findings were compatible with a diffuse infiltration of the lungs by NPDB, also confirmed by the results of pulmonary functional tests (PFTs) (predominantly restrictive pattern with signs of obstruction of the small, distal airways, severe reduction of gas diffusion through alveolarcapillary membrane, abnormal oxygen desaturation at six minutes walk test) and bronchoalveolar lavage (BAL) ("foamy" histiocytes with their cytoplasm almost completely occupied by swollen lysosomes containing pleomorphic inclusions variable in size and electrondensity, a finding consistent with Niemann-Pick cells) (NPCs).

\section{Discussion}

Niemann-Pick disease is a rare lysosomal storage disorder characterised by SM accumulation within the cells of the reticuloendothelial system, mainly but not exclusively located in the spleen, the liver and the bone marrow; it is a heterogeneous biochemical and clinical entity, currently divided into six subtypes, from $\mathrm{A}$ to $\mathrm{F}$, with variable extent and severity depending on the underlying genetic alterations and other exogenous factors [1, 2]. A mutation in the gene SPMD1, encoding the ASM, together with less important derangements of enzymatic and non-enzymatic proteins involved in lysosomal metabolism and intracellular cholesterol trafficking and processing, is associated with the two most frequent subtypes of the disease, A (classic, infantile form) and B (chronic, polivisceral, non-neuropathic form); a mutation in the gene NPC1 is associated with subtypes $C$ (subacute, juvenile form) and D (Nova Scotian form), while still poorly understood is the etiology of subtypes $\mathrm{E}$ and $\mathrm{F}$, rare and less frequently described in literature [1, 3, 4]. Conversely from what occurs in NPDA, characterised by almost complete absence of ASM activity, severe and rapidly progressive involvement of the central nervous system, diagnosis in the first weeks of life, and early exitus in childhood in most of the cases, NPDB is generally associated with a certain residual enzymatic activity, leading to a lat- er diagnosis, a chronic, slowly but inexorably progressive polivisceral involvement with spur of the nervous system and survival to adulthood in most of the cases. First manifestations of NPDB are generally related to the involvement of the spleen (massive splenomegaly), the liver (mild hepatomegaly) and the bone marrow (mainly thrombocytopenic purpura) being the reticuloendothelial cells mainly located in these anatomical districts; later may appear general malaise, fatigue, endocrine, metabolic and emocoagulative disorders (e.g. growth retardation, delayed sexual maturity, dyslipidemya, deficit of several coagulation factors), anaemia and leukopenia, skin pigmentation, malabsorption syndromes and retinal changes. Several authors have reported the association between lysosomal storage disorders (e.g. Gaucher's disease and Fabry disease) and valvular disease; Minai and coll., in particular, described the case of a 48 year-old patient with pulmonary involvement by NPDB operated three years before their report for aortic valve replacement [5], as happened in our patient. It is not yet clear whether there is a correlation between the two conditions and, in particular, it is still unknown the mechanisms through which storage disorders could lead to thickening, chondroid degeneration and calcification of the valve leaflets, all findings widely described in literature (e.g. direct deposition of the metabolite or of the cells containing it, induction of integrin or complement-mediated proliferative and inflammatory pathways). What is certain, in our opinion, is that the presence of three valvular abnormalities (aortic valve stenosis, mitral stenoinsufficiency and tricuspidal insufficiency) in our relatively young patient cannot be simply interpreted as a coincidence with NPDB but should be considered as a consequence of the metabolic disorder until proven otherwise [5-7]. The prognosis of NPDB is discrete with survival to adulthood in most cases; exitus usually occurs because of spleen rupture, severe bleeding due to coagulation disorders, liver failure and, most importantly, onset of severe respiratory complications $[4,5,8-10]$. Pulmonary involvement may virtually occur at any time in NPDB, although it is rarely its first manifestation, generally appearing at a relatively advanced phase of the disease in the adulthood, preceded by signs and symptoms related to the involvement of other districts and then evolving with a slowly and inexorably progressive course to the point of assuming greater prognostic importance over time by determining severe respiratory failure, pulmonary hypertension, or infectious complications [5]. Although lung involvement is an important cause of morbidity and mortality in adult patients affected by NPDB, the variant most frequently associated with respiratory manifestations among the six described to date, its exact incidence is difficult to determine for several reasons:

- in adults, as in childhood and adolescence, lung involvement in NPD has been relatively little studied compared to that of other organs, particularly the central nervous system and the liver [11];

- unlike what happens in childhood, the incidental radiographic finding of lung infiltration in 

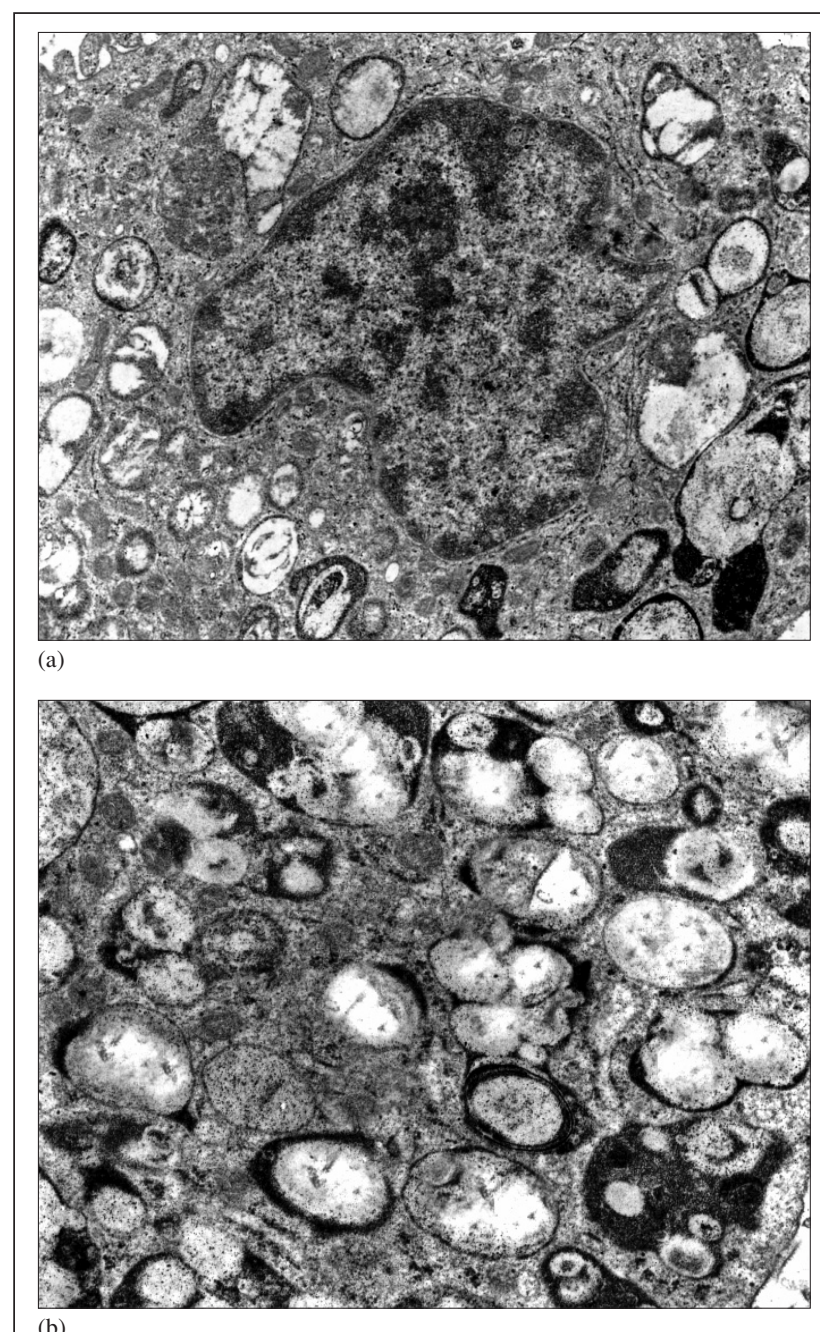

(b)

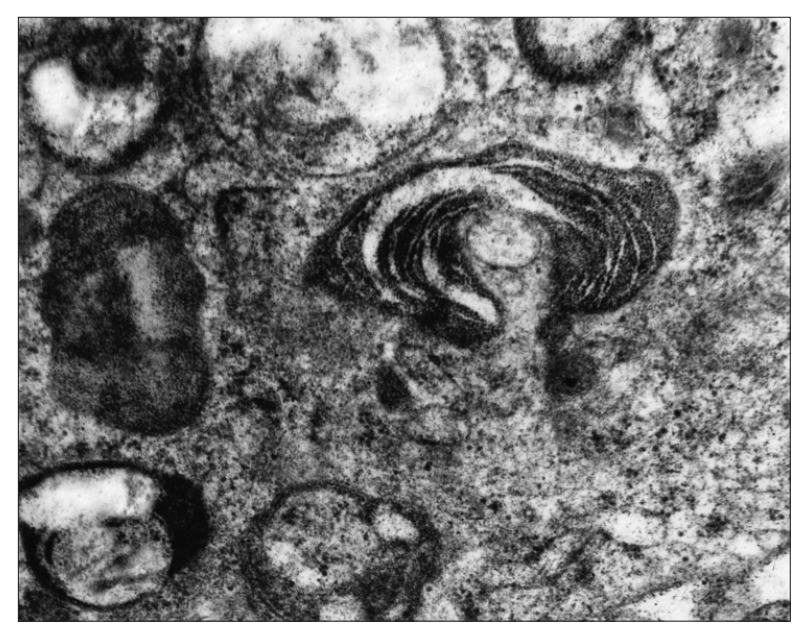

(c)

Fig. 1 a, b, c. - Electron microscopy findings in our patient. a) A macrophage with indented nucleus and cytoplasm containing many inclusions and some pleomorphic mitochondria $(7.000 \mathrm{x})$. b) Pleomorphic cytoplasmic inclusions of varying size and electrondensity $(12.000 \mathrm{x})$. c) Clear and electrondense inclusions surrounded by concentric lamellar structures $(20.000 \mathrm{x})$.

absence of other signs of disease in an adult is rarely recognised as a manifestation of a storage disease (especially if not previously diagnosed) and most often handled as a generic respiratory infection; the course of the disease, usually very mild, spontaneously evolves towards the disap- pearance of symptoms over several days, thus supporting the wrong diagnoses;

- many forms run completely asymptomatically, thus escaping the scientific report, unless they are incidentally discovered through the execution of laboratory or imaging examinations (mainly CXRs) performed for other reasons [2];

- most authors like us, finally, do not accept the idea of an "adult-onset" pulmonary involvement in NPDB, merely considering the appearance of respiratory manifestations in an adult affected as an epiphenomenon of histopathological changes already started during the first weeks or months of life, then slowly and insidiously progressed until reaching a certain degree of parenchymal impairment during the childhood and the adolescence only causing mild and nonspecific symptoms (e.g. recurrent respiratory infections) so not to be considered by the pediatrician or the parents or not causing any symptom at all. This clinical and, as described further, functional and radiographic "evolutivity" (strongly influenced in terms of severity and rapidity of progression by the type of SMPD1 mutation and, consequently, the degree of residual ASM activity, the association with respiratory infections, cigarette smoking and other diseases, particularly diabetes mellitus [4, 5, 9, 10, 12-14] reflects the progressive infiltration of the interstitium, the alveolar spaces, the tracheobronchial walls, the peribronchial and subpleural lymphatic spaces by foamy, lipid-laden macrophages, also indicated as NPCs, showing typical features at optical (multivacuolated cytoplasm containing granules intensely sea-blue staining at May-GrunwaldGiemsa technique, belonging to the "sea-blue histiocytosis", an heterogeneous group including other thesaurismosis and hemopathies $[4,15,16]$ and electron (swollen lysosomes containing clear vacuoles and electrondense granules, the latter corresponding to lipofuscin and ceroid deposits [17]) microscopy (fig. $1 \mathrm{a}, \mathrm{b}, \mathrm{c}$ ). Since the early days of life lungs are normally settled by two histiocytic populations, interstitial and alveolar macrophages which, although originating from a common ancestor in the bone marrow, then play different roles, locating the first in the interlobular and intralobular septa, where they take part in non-specific and specific immune responses, the latter in the alveolar spaces, involved as well as in immune defense mechanisms in the metabolism of surfactant; the impairment of the latter two vital processes explains the frequent appearance of respiratory failure syndromes and respiratory infections, in particular lipoid pneumonias, in NPDB $[1,3,5,9$, 13-21].

The progressive histiocytic infiltration of interlobular septa and alveolar spaces causes the appearance and the increase over time of the two most frequent findings at CXR in adult patients affected by NPD, particularly subtype B: reticulonodular interstitial markings, sometimes creating a "honeycombing" pattern even in the absence of fibrosis and associated with Kerley B lines [22], and "patchy" or "geographically" - distributed "ground glass" opacities, the latter generally appearing in a 
relatively more advanced phase of the disease [13, $15,23]$; the involvement of intralobular septa is generally less important, still minimally contributing to both patterns [15] (fig. 2). Low sensitivity and specificity, the inherent limitations of CXR in the evaluation of interstitial lung disease, have been overcome by the advent of HRCT, which confirms and better defines the extent of the two above-mentioned nonspecific findings and adds new, more specific ones through a detailed visualization of parenchymal anatomy to the level of the secondary lobule. Thickened interlobular septa and "ground glass" opacities tend to initially appear at lung bases, then slowly cranially progressing [20] (fig. 3); in the advanced stages of the disease, however, these two findings involve surprisingly homogeneously and regularly both lungs at HRCT, so reflecting the systemic involvement of NPDB [16]. In some areas septal reticulonodular thickening and "ground glass" opacities intermix creating a "crazy paving" pattern (fig. 4), not specific for NPD, initially reported in a series of cases of alveolar proteinosis and also described in bronchioloalveolar carcinoma, sarcoidosis, pneumocystis carinii pneumonia, exogenous lipoid pneumonia, Wegener's granulomatosis, ChurgStrauss syndrome, Goodpasture's syndrome and other thesaurismosis [24]. Calcified and non-calcified nodules may also be observed (fig. 4), the latter being characterised by hazy margins and predominantly centrilobular distribution and sometimes difficult to appreciate at HRCT because of the superimposed thickened septa and "ground glass" opacities): they are generally less than one centimeter in diameter and correspond to fatty deposits within the bronchial and bronchiolar epithelial cells, where ASM plays a pivotal role in normal conditions, being involved in the processes of oxidative metabolism. Pleural abnormalities, lung cysts and lymph nodal enlargement are very rare findings $[2,13,15,21,23-26]$. Gaucher's disease, Gangliosidoses, Fabry disease [27], Hermansky-Pudlak syndrome, pulmonary oedema, linfomatous or, less frequently, carcinomatous lymphangitic spread [20], proteinosis and

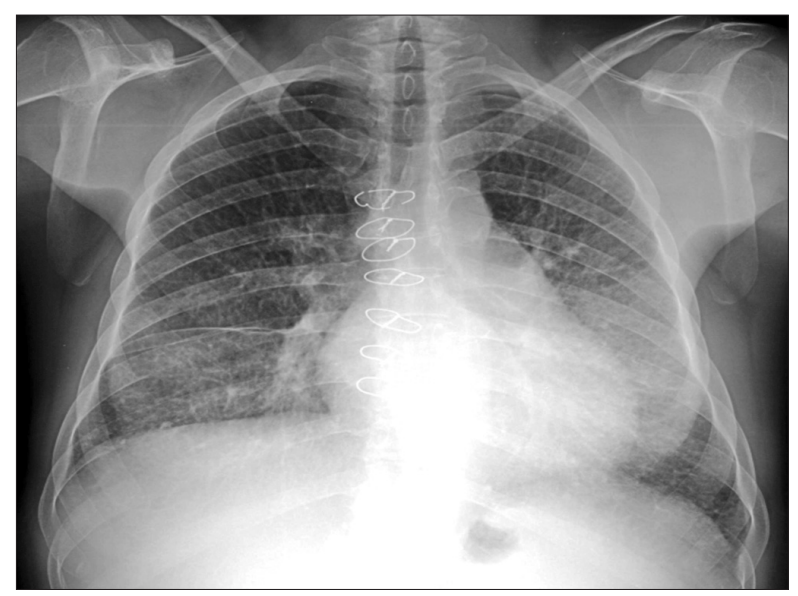

Fig. 2. - Chest X-Ray abnormalities in our patient. Bilateral reticulonodular interstitial thickening prevalent al lower lobes, thickened minor fissure, scattered "ground glass" opacities, mainly in the left lung. alveolar microlithiasis, desquamative interstitial pneumonia, lymphoid interstitial pneumonia, nonspecific interstitial pneumonia [9], miliary tuberculosis and amyloidosis [24] are all conditions with a HRCT pattern similar to that of NPDB, resulting in a difficult differential diagnosis, often requiring an histopathological confirmation, especially when sign or symptoms related to the involvement of other organs lack and the storage disease has not previously been diagnosed. This confirmation, as already mentioned in the introduction, is generally obtained by BAL, a moderately invasive procedure based on the passage of fluid through a bronchoscope into the lower airways and its subsequent recollection for the cytohistological exam. NPD is one of the few conditions where whole-lung lavage is recommended. This technique is useful both in terms of diagnosis and therapy in NPD as in other conditions (alveolar proteinosis, severe dyspnea or hypoxemia, $\mathrm{PaO}_{2}$ at sea level of about $65 \mathrm{mmHg}$ and alveolar-arterial oxygen tension gradient of about $40 \mathrm{mmHg}$ or a shunt fraction exceeding $10 \%$ to $12 \%)$ : the identification of the pathognomonic "foamy" histiocyte at optical and electron microscopy, in fact, leads to the rare and difficult definitive diagnosis. Furthermore, whole-lung lavage results in significant symptomatic and radiographic improvement by reducing the surfactant impairment and the alveolar filling due to the presence of foamy cells.

The same way as the bilateral interstitial thickening observed at CXR and HRCT, the abnormalities of PFTs slowly and steadily progress over time in NPDB: pulmonary flows and lung volumes remain generally unchanged until relatively advanced stages of the disease; later a worsening restrictive pattern associated with signs of obstruction of the small, distal airways appears. The progressive septal infiltration and alveolar concentration by NPCs causes abnormalities of gas exchange through the alveolar-capillary membrane (moderate-severe reduction of DLCO values in the later stages of disease) associated with the pathologic oxygen desaturation observed at

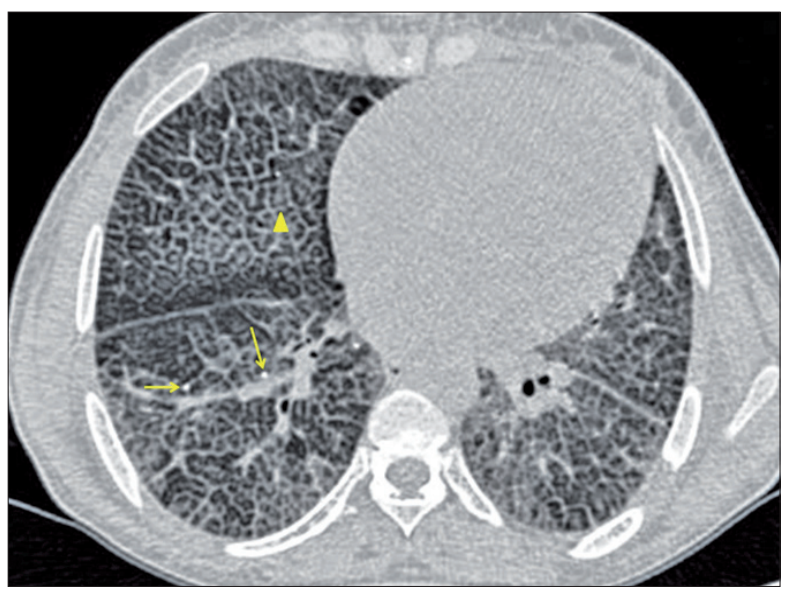

Fig. 3. - Progression of lung injury in NPDB. Septal thickening and "ground glass" opacities are initially prevalent at lung bases, then cranially progressing up to cover the whole lungs. 


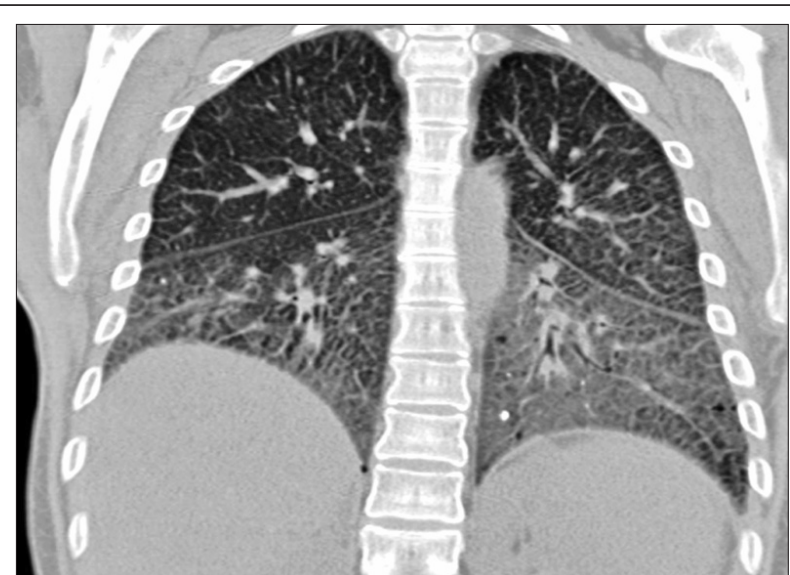

Fig. 4. - "Crazy paving" pattern created by the intermixing of ground glass opacities and thickened interlobular septa; several millimetric calcific nodules and a hazy, centrilobular non calcific nodule are also appreciable.

the six minutes walking test in the patients affected by NPDB [5, 28]. Unlike what happens in most of the interstitial lung diseases (ILDs), especially in idiopathic pulmonary fibrosis, characterized by a predictable correlation between radiographic abnormalities and functional impairment throughout the whole duration of the disease, in the early stages of NPDB there is a typical, remarkable "disassociation" between radiographic and functional findings due to the histopathological substrate of septal thickening: although interstitial infiltration by the lipid-laden histiocytes causes the appearance of CXR and HRCT patterns similar to those observed in fibrosing ILDs, there is no ultrastructural remodelling of the "only congested" alveolar-capillary membrane, almost entirely lacking fibrosis and other signs of chronic involvement, with DLCO values amazingly unchanged even in the presence of marked radiographic signs $[5,14,20]$. It is also possible, however, to observe absent or mild radiographic abnormalities in association with a severe respiratory failure, resulting in the need to integrate the results of functional tests and radiographic exams in order to achieve a proper balance of the disease [13]. Another factor not to be overlooked in the occurrence of respiratory failure in patients with NPDB, probably also significant in the case we described, is hepatosplenomegaly, sometimes marked so as to interfere with diaphragmatic activity [13].

\section{References}

1. Crocker AC, Farber S. Niemann-Pick disease: a review of 18 patients. Medicine 1958; 37: 1-98.

2. Falco F, Bembi B, Cavazza A, Pittis MG, Zucchi L. Pulmonary involvement in an adult female affected by type B Niemann-Pick disease. Sarcoid Vasc Diff Lung Dis 2005; 22: 229-33.

3. Dhami R, He X, Gordon RE, Schuchman EH. Analysis of the lung pathology and alveolar macrophage function in the acid sphingomyelinase deficient mouse model of Niemann-Pick disease. Lab Invest 2001; 81: 987-99.

4. Long RG, Lake BD, Petit JE, Scheuer PJ, Sherlok S. Adult Niemann-Pick disease. Its relationship to syn- drome of the blue sea histiocytes. Am J Med 1977; 62: 627-35.

5. Minai OA, Sullivan EJ, Stoller JK. Pulmonary involvement in Niemann-Pick disease: case report and literature review. Resp Med 2000; 94; 1241-51.

6. Guertl B, Noehammer C, Hoefler G. Metabolic cardiomyopaties. Intern J Exp Path 2000; 81: 349-72.

7. Veinot JP, Elstein D, Hanania D, Abrahamov A, Srivatsa S, Zimran A. Gaucher's disease with valve calcification: possible role of Gaucher cells, bone matrix proteins and integrins. Can J Cardiol 1999; 15: 211-6.

8. Lever AML, Ryder JB. Cor pulmonale in an adult secondary to Niemann-Pick disease. Thorax 1983; 38: 873-4.

9. Nicholson AG, Florio R, Hansell DM, du Bois RM, Wells AU, Hughes P, Ramadan HK, Mackinlay CI, Brambilla E, Ferretti GR, Erichsen A, Malone M, Lantuejoul S. Pulmonary involvement by Niemann-Pick disease. A report of six cases. Histopathol 2006; 48: 596-603.

10. Nicholson AG, Wells AU, Hooper J, Hansell DM, Kelleher A, Morgan C. Successful treatment of endogenous lipid pneumonia due to Niemann-Pick type B disease with whole-lung lavage. Am J Resp Crit Care Med 2002; 165: 128-131.

11. Guillemot N, Troadec C, Bilette de Villemeur T, Clement A, Fauroux B. Lung disease in Niemann-Pick disease. Pediatr Pulmon 2007; 42: 1207-14.

12. Gonzalez-Reimers E, Sanchez-Perez MJ, Bonilla-Arjona A, et al. Pulmonary involvement in an adult male affected by type B Niemann-Pick disease. Br J Radiol 2003; 76: 838-40.

13. Mendelson DS, Wasserstein MP, Desnick RJ, et al. Type B Niemann Pick disease: findings at chest radiography, thin-section CT and pulmonary function testing. Radiology 2006; 238: 339-45.

14. Wasserstein MP, Desnick RJ, Schuchman EH, Hossain S, Wallenstein S, Lamm C, Mc Govern MM. The natural history of type B Niemann-Pick disease: results from a 10-year longitudinal study. Pediatrics 2004; 114: 672-7.

15. Hervè A, Marchand-Adam S, Fabre A, et al. Maladie de Niemann-Pick de type B revelee par une atteinte bronchopulmonaire. Rev Mal Respir 2008; 25: 861-6.

16. Piercecchi MD, Sault MC, Cailleres S, Blanc AP, Sudan N. Pneumopathie interstitielle rivelatrice d'une maladie de Niemann-Pick de type B chez un adulte. Rev Med Interne 1999; 20: 597-601.

17. Skikne MI, Prinsloo I, Webster I. Electron microscopy of lung in Niemann-Pick disease. J Pathol 1972; 106: $119-22$.

18. Muntaner L, Galmes A, Chabas A, Herrera M. Imaging features of type B Niemann-Pick disease. Eur Radiol 1997; 7: 361-4.

19. Marten K, Hansell DM. Imaging of macrophage-related lung disease. Eur Radiol 2005; 15: 727-41.

20. Ferretti GR, Lantuejoul S, Brambilla E, Coulomb M. Case report. Pulmonary involvement in Niemann-Pick disease subtype B: CT findings. J Comput Assist Tomogr 1996; 20: 990-2.

21. Rodrigues R, Marchiori E, Muller NL. Niemann-Pick disease. High-resolution CT findings in two siblings. $J$ Comput Assist Tomogr 2004; 28: 52-4.

22. Duchateau F, Dechambre S, Coche E. Imaging of pulmonary manifestations in subtype B of Niemann-Pick disease. Br J Radiol 2001;74: 1059-61.

23. Lachman R, Crocker A, Schulman J, Strand R. Radiological findings in Niemann-Pick disease. Radiology 1973; 108: 659-664.

24. Rossi SE, Erasmus JJ, Volpacchio M, Franquet T, Castiglioni T, Mc Adams P. "Crazy paving" pattern at thinsection CT of the lungs: radiologic-pathologic overview. Radiographics 2003; 23: 1509-19.

25. Baldi BG, Santana ANC, Takagaki TY, Fujita C, 
Kairalla RA, Carvalho CRR. Lung cysts: an unusual manifestation of Niemann-Pick disease. Respirology 2009; 14: 134-6.

26. Bouziani A, Cheikh R, Ben Rejeb A, et al. Manifestation tomodensitometrique thoracique et cytologie du liquide delavage broncho-alveolaire dans un case adulte de maladie de Niemann-Pick. Rev Mal Resp 1990; 7: 267-71.
27. Chung MJ, Lee KS, Franquet T, Muller NL, Han J, Kwon OJ. Metabolic lung disease: imaging and histopathologic findings. Eur J Radiol 2005; 54: 233-45.

28. Mc Govern MM, Wasserstein MP, Giugliani R, et al. A prospective, cross-sectional survey study of the natural history of Niemann-Pick disease. Pediatrics 2008; 122: 341-9.

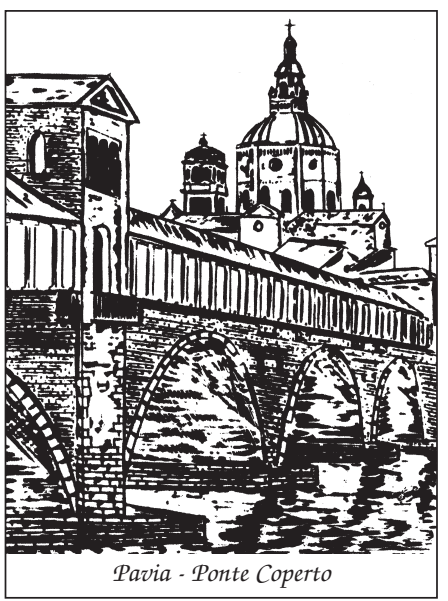

